\title{
AXIAL GAS TRANSPORTS IN DC-DISCHARGES IN MIXTURES OF GASES
}

\author{
by J. FREUDENTHAL*) \\ lysisch Laboratorium der Rijksuniversiteit te Vtrecht, Nederland
}

\section{Synopsis}

Selective transports in DC-discharges in gas mixtures cause a cataphoretic segregation of the gas mixture. The two transports treated are: 1) the ion transport and 2) the transport due to the momentum transfer of the electrons and ions to the gas. A stationary state will occur as a result of the counteracting diffusion transport. The density distribution of the gases along the positive column is derived as a function of time, and in the stationary state.

Introduction. It is a well-known fact that a DC-discharge in a mixture of gases will cause an axial segregation of the gases $\left.{ }^{1}\right)^{2}$ ). This phenomenon is often called "cataphoretic segregation" 3-14). For example, in spectrochemical micro-analysis, the segregation is used to concentrate the traceelements to be analysed on the cathode side of the arc $\left.{ }^{1.5-18}\right)$. In the following we shall confine ourselves to the discussion of the effect in the positive column of a DC-discharge at pressures of a few torr. Sometimes this effect is that the concentration of one of the gas components will vary strongly along the positive column. Then the colour of the discharge on the cathode side may differ markedly from that on the anode side.

Selective gas transports are responsible for the segregation. The mechanism of the gas transports occurring in the DC-discharge is described below. Although the description is valid for a multi-component gas mixture we will confine ourselves to a gas mixture of two components 1 and 2, with densities**) of $n_{1}$ and $n_{2}$, respectively.

Moreover we shall suppose that, besides the electrons, there are only positive ions***)

*) Present address: University of Minnesota, Dept. Electr. Engineering Minneapolis, Minnesot a U.S.A.

**) The was density used refers to the number of atoms per unit of volume.

***) In gas discharges where negative ions are present too, a corresponding extra transport term for the negative ions has to be introduced. Then the derivation of the formulas for the gas transports is analogous to that in gas discharges with positive ions only. 
1. Ion transports in the discharge tube. The positive ions of for example component 1 have an axial drift velocity $v_{1}^{+}$in the axial electric field $(E)$. The drift velocity $v_{1}^{+}$can be written as $v_{1}^{+}=\mu_{1}^{+} E$, by which $\mu_{1}^{+}$is defined as the ion mobility of the ions of component 1 in the gas mixture. The transport density of the ions of component $1, \Gamma_{1}$, is given by:

$$
\Gamma_{1}=n_{1}^{+} \mu_{1}^{+} E .
$$

The ion transport of component 1 is equivalent to a gas transport. The transport density $\Gamma_{1}$ is in the direction of the cathode. Gas component 1 will be enriched on the cathode side of the discharge tube. Consequently component 1 will diffuse in the opposite direction. The diffusion transport density $\Gamma_{1}^{*}$ is:

$$
\Gamma_{1}^{*}=D \frac{\mathrm{d}}{\mathrm{d} x} n_{1},
$$

with $D$ as the mutual diffusion coefficient in the gas mixture and $(\mathrm{d} / \mathrm{d} x) n_{1}$ as the axial gradient of the density of gas component 1 . In the diffusion transport density $\Gamma_{1}^{*}$, the diffusion of the ions is neglected. This approximation is allowed when the ionization degree is low. Generally, the ionization degree of each of the gas components is low; in the experiments described in the next paper ${ }^{2}$ ) this was the case too.

In the stationary state the transports given in the formulas (1) and (2) are equal ${ }^{6}$ ) (assuming these two types of transports only):

$$
n_{1}^{+} \mu_{1}^{+} E=D \frac{\mathrm{d}}{\mathrm{d} x} n_{1} .
$$

2. Gas transports due to momentum transfer. Let $n^{-}$be the electron density and $E$ the axial field, then the field will give a momentum per second per unit of volume of $n^{-} e E$, this momentum being given by the electrons to the gas atoms. A same amount of momentum is transferred to the gas by the positive ions, but in the opposite direction compared to that of the electrons (as the plasma is quasi-neutral)*).

Let us assume a discharge in a mixture of two gases containing positive ions (densities $n_{1}^{+}$and $n_{2}^{+}$) and electrons (density $n^{-}$). The plasma is quasineutral, and if we use $n^{+}$for $n_{1}^{+}+n_{2}^{+}$then $n^{+} \approx n^{-}$. The electric field gives the positive ions a momentum of $n^{+} e E$ in the direction of the cathode and the electrons a momentum of $n^{-} e E$ in the direction of the anode. The net transfer of momentum on the gas is approximately zero and no force will act on the gas. This does not mean that no force is acting on each of the gas components.

*) Actually the momentum transfer by the electrons is somewhat greater than the momentum transfer by the ions (electrophoresis). However, at total gas pressures of a few torr and tube diameters of a few $\mathrm{cm}$ the effect is negligible ${ }^{27}$ ). 
Generally, the cross sections for momentum transfer from the electrons to the gas atoms and from the ions to the gas atoms are different for the gas components and as a consequence there will be a net amount of momentum transferred to each of the gas components. Let $P_{1}$ be the net transferred momentum per unit of volume and per second by the electrons and ions to component 1 ; this is equivalent to a force acting on gas component 1 and the resulting axial gradient is ${ }^{19}$ ):

$$
\stackrel{\mathrm{d}}{\mathrm{d} x} n_{1}=\frac{P_{1}}{k T},
$$

Where $P_{1} \sim n_{1}$ and with $k$ as the Boltzmann constant and $T$ as the gas temperature.

For the calculation of $P_{1}$, one has to consider the momentum transfer by the electrons and ions separately.

The total amount of momentum transferred by the electrons as well as the ions, $n^{-} e E$, will be rewritten by substituting

$$
n^{-}=\frac{J}{c \mu^{-} E} \quad \text { and } \mu^{-}-\frac{M_{1,2}^{-}}{p_{1}+p_{2}},
$$

where $M_{1,2}^{-}$is the reduced electron mobility (at one torr pressure) in the gas mixture, and where $p_{1}$ and $p_{2}$ are the partial pressures of the two gas components. This gives:

$$
n^{-} e E=\frac{J}{\mu^{-}}=\frac{p_{1}+p_{2}}{M_{1,2}} J
$$

The distribution of the momentum transferred in the axial direction over the two gas components is proportional to the cross sections for momentum transfer. The cross sections for momentum transfer can be identified with the diffusion cross sections describing the diffusion coefficient and mobility $\left.{ }^{19}\right)^{20}$ ). The distribution of the momentum transferred by the electrons over the two gas components is in accordance with the partial pressures and the reduced mobilities (compare the law of Blanc ${ }^{21}$ ): on gas component 1 :

and on gas component 2 :

$$
\underset{M_{\mathrm{t}}^{-}}{J} p_{1}
$$

$$
\frac{J}{M_{2}} \cdot p_{2}
$$

where $M_{1}^{-}$and $M_{2}^{-}$are the reduced electron mobilities in the gas components 1 and 2 respectively at the elcctrons tempcrature occurring in the gas mixture.

For the calculation of the momentum transferred by the ions to gas 
component 1 , we have to consider the momenta transferred by both types of ions (densities $n_{1}^{+}$and $n_{2}^{+}$). The total momentum transferred by the ions of type 1 is

$$
\frac{n_{1}^{+}}{n^{+}} \frac{J}{M_{1,2}^{-}}\left(p_{1}+p_{2}\right),
$$

(and does not depend on the type of the ion). The momentum transferred to gas component 1 is again determined by the partial pressures and the reduced mobilities:

$$
\frac{p_{1} / M_{1-1}^{+}}{p_{1} / M_{1-1}^{+}+p_{2} / M_{1-2}^{+}} \frac{n_{1}^{+}}{n^{+}} \frac{J}{M_{1,2}^{-}}\left(p_{1}+p_{2}\right),
$$

where $M_{i-j}^{+}$is the reduced mobility of ion type $i$ in gas type $j$. In an analogous way the momentum transferred by the ions of type 2 to gas component 1 is:

$$
\frac{p_{1} / M_{2-1}^{+}}{p_{1} / M_{2-1}^{+}+p_{2} / M_{2-2}^{+}} \frac{n_{2}^{+}}{n^{+}} \frac{J}{M_{1,2}^{-}}\left(p_{1}+p_{2}\right) \text {, }
$$

The total force on gas component 1 is the sum of the forces given in the formulas (6), (7) and (8). (The force given in formula (6) is directed towards the anode and the forces given in the formulas (7) and (8) are directed towards the cathode). When $p_{1} \ll p_{2}$ and when nearly all ions are ions of the type 1 (for instance in neon (comp. 2) with $1 \%$ argon (comp. 1) ${ }^{2}$ ), then the total force on gas component 1 follows from the formulas (6) and (7):

$$
\left[\frac{M_{1-2}^{+}}{M_{1-1}^{+}}-\frac{M_{1,2}^{-}}{M_{1}^{-}}\right] \frac{J}{M_{1,2}^{-}} p_{1} .
$$

In the case that $p_{1} \ll p_{2}$, but that $n_{1}^{+} \ll n_{2}^{+}$(for instance neon with a very small amount of $\left.\operatorname{argon}^{2}\right)$ the force on gas component 1 follows from the formulas (6) and (8).

$$
\left[\frac{M_{2-2}^{+}}{M_{2-1}^{+}}-\frac{M_{1,2}^{-}}{M_{1}^{-}}\right] \frac{J}{M_{1,2}^{-}}-p_{1} \text {. }
$$

The relative importance of the two transports can be seen from the ratio between the forces causing these transports. This ratio will be considered for two cases:

a. $n_{1}^{+} \gg n_{2}^{+}$and $p_{1} \ll p_{2}$; the force on the ions (comp. 1) caused by the electric field is:

$$
n_{1}^{+} e E \approx n^{+} e E=\frac{J}{M_{1,2}^{-}} p_{2} .
$$

Then the ratio between the force of the electric field on the ions and the momentum transfer of the ions and electrons on the gas is given by:

$$
\frac{p_{1}}{p_{2}}\left[\frac{M_{1-2}^{+}}{M_{1-1}^{+}}-\frac{M_{1,2}^{-}}{M_{1}^{-}}\right] \text {. }
$$


b. $n_{1}^{+-} n_{2}$ and $p_{1} \leqslant p_{2}$; the ratio between the forces is in this case compare formula (8)):

$$
\begin{array}{ll}
p_{1} & n^{+} \\
p_{2} & n_{1}^{-}
\end{array}\left[\begin{array}{ll}
M_{2-2}^{+} & M_{1,2}^{-} \\
M_{2-1}^{+} & M_{1}^{-}
\end{array}\right] .
$$

It follows from the formulas (11a) and (11b) that the influence of the transport by momentum transfer by the ions and electrons is small in the case that $p_{1} \ll p_{2}$ and when the gas of component 1 (admixture gas) can be ionized relatively strong $\left(n_{1}^{+} / n^{+}\right.$is large). These types of gas mixtures will be considered in the next pages and in the following paper ${ }^{2}$ ).

3. The axial distribution of the gas in the stationary state. The density distribution of the gases along the positive column will be considered in the case that the ion transport is the main cause of the segregation. Transports due to momentum transfer of the electrons and ions are neglected. This simplification is possible in experimental studies where use is made of certain mixtures ${ }^{2}$ ). As was shown this type of mixtures always contains a main gas (density $n_{m}$ ), and an admixture gas which can be ionized relatively easily (density $n_{a}$ ).

The distribution of the admixture $n_{a}(x)$ depends on $\alpha$, where $\alpha$ is the ratio between $n_{a}^{+}$and $n^{+}\left(n^{+}=n_{a}^{+}+n_{m}^{+}\right)$. We shall distinguish three cases for $\alpha$ : namely $\alpha=1$ (thus $\left.n_{m}^{+} \ll n_{a}^{+}\right), \alpha$ is small $\left(n_{a}^{+}<n_{m}^{+}\right)$, and a value for $\alpha$ between the two cases mentioned.

a. Nearly all ions are admixture ions $(\alpha \approx 1)$. In the stationary state the ion transport density is equal to the diffusion transport density, and according to formula (3):

$$
n_{t}^{+} \mu_{a}^{+} E-D \frac{\mathrm{d}}{\mathrm{d} x} n_{a} .
$$

When all ions are admixture ions, $n_{u}^{+}=n^{+}$. In a quasi neutral plasma $n^{+} \approx n^{-}$, with $n^{-}$as the electron density.

Equation (12) may be rewritten by substituting for $n_{a}^{+}$other gas discharge parameters.

$$
n_{a}^{+} \approx n^{-}=\frac{J}{e \mu^{-E}},
$$

with $\mu^{-}$as the electron mobility, $J$ as the tube current density and $E$ as the field strength. Substitution of formula (13) in (12) gives:

$$
\frac{\mu_{a}^{+}}{\mu^{-}} \frac{J}{e}=D \frac{\mathrm{d}}{\mathrm{d} x} n_{a} \text {. }
$$

The distribution $n_{a}(x)$ may be obtained by integration of formula (14).

$$
n_{a}(x)=n_{a}(0)-c x,
$$


with $c \equiv \mu_{a}^{+} J / \mu^{-} D e$, supposing $c$ does not depend on $x$. The density of the admixture in $x=0$ is denoted by $n_{a}(0)$. The location of the cathode is called $x=0$.

In discharges where the pressure and gas temperature are uniform it can be shown that the quantities appearing in $c$ remain constant (or may change only to a less extent). This means that we will find a linear distribution for this case of cataphoresis.

The function $n_{a}(x)$ given in formula (15) has to be normalized to $n_{a}^{*}$ (the average density of the admixture). Then the density $n_{a}(0)$ has to be chosen such that

$$
\int_{0}^{L} n_{a}(x) \mathrm{d} x=n_{a}^{\star} L,
$$

with $L$ as the length of the discharge tube.

$b$. A small fraction of the ions are admixture ions $(\alpha$ is small). If $\alpha$ is small the ratio $n_{a}^{+} / n_{m}^{+}$will be small. Then $n_{a}$ is very much smaller than $n_{m}$.

When in the destruction process of the admixture ions no admixture atoms are involved the density of the admixture ions $\left(n_{a}^{+}\right)$will be proportional to the density of the admixture itself assuming a constant electron temperaturc. This can be written as:

$$
n_{a}^{+}=\theta n_{a},
$$

in which the constant of proportionality $\theta$ is the ionization degree of the admixture gas. Substitution of formula (16) in (12) gives:

$$
\theta n_{a} \mu_{a}^{+} E=D \frac{\mathrm{d}}{\mathrm{d} x} n_{a}
$$

The solution of equation (17) is:

$$
n_{a}(x)=n_{a}(0) \exp \left[-\frac{\mu_{a}^{+}}{D} E \theta x\right],
$$

where $n_{a}(0)$ is the density at the cathode $(x=0)$ of the discharge tube. Normalization of formula (18) gives:

$$
n_{a}(x)=n_{a}^{*} \frac{u L}{D} \frac{1}{1-\mathrm{e}^{-u L / D}} \exp \left[-\frac{u x}{D}\right],
$$

wherc $n_{a}^{*}$ is the average density of the admixture and $u \equiv \mu_{a}^{+} E \theta$. A normalization according to formula (19) is applicable in the case that the cathode and anode are mounted at the ends of the discharge tube.

c. An arbitrary fraction of the ions are admixture ions. For arbitrary of $\alpha(0<\alpha<1)$ we can state only

$$
n_{a}^{+}=\alpha n^{+} \approx \alpha n^{-}=\frac{\alpha J}{e \mu^{-} E} .
$$


In cases when $\alpha$ is independent of $x$, substitution of formula (20) in (11) and integration of the result gives:

$$
n_{a}(x)=n_{a}(0)=c^{\prime} x
$$

where $c^{\prime} \equiv \mu_{a}^{+} \alpha J / \mu^{-} D e$. The distribution function $n_{a}(x)$ may be normalized. However, in many cases $\alpha$ will be a function of $n_{a}$ and thus a function of $x$, and consequently $n_{a}$ will be a more complicated function of $x$.

4. Influence of radial effects on axial gas transports. The derivation of formula (11): $n_{a}^{+} \mu_{a}^{+} E=D(\mathrm{~d} / \mathrm{d} x) n_{a}$ was a treatment for a one-dimension problem. However, $n_{a}^{+} \cdot \mu_{a}^{+}, D$, and $(\mathrm{d} / \mathrm{d} x) n_{a}$ are functions of $x$ and $r$ (with $r$ as the distance to the axis of the discharge tube). The axial field strength is constant over the cross section of the tube. The total ion transport is:

$$
2 \pi E \int_{n}^{l} n_{a}^{+\cdot}(r) \mu_{\| !}^{+}(r) r \mathrm{~d} r,
$$

and the total diffusion transport is:

$$
2 \pi \int_{0}^{l} D(r) \cdot \frac{\mathrm{d}}{\mathrm{d} x} n_{a}(r) r \mathrm{~d} r .
$$

In the stationary state the two transports are equal:

$$
2 \pi E \int_{0}^{R} n_{n}^{r}(r) \mu_{t}(r) r \mathrm{~d} r-2 \pi \int_{0}^{R} D(r) \mathrm{d} n_{a}(r) r \mathrm{~d} r .
$$

To calculate the integrals (22) and (23) the functions $n_{t}(r), \mu_{i t}^{-i}(r), D(r)$ and $(\mathrm{d} / \mathrm{d} x) n_{a}(r)$ must be known.

a. The ion density $n_{a}^{+}$as a function of $r$. As a result of the ambipolar diffusion of the electrons and ions to the wall of the tube a distribution of the density of the electrons in the radial direction will appear. The functions $n^{-(}(r)$ and $n^{+}(r)$ are nearly the same (quasi-neutrality of the plasma). Schot $\mathrm{ky}^{22}$ ) has derived the dependence in the case of a small mean free path of the electrons compared to the radius $R$ of the discharge tube. If we assume $n_{a}^{+}=n^{+}$, then the distribution $n_{a}^{+}(r)$ is given by:

$$
n_{a}^{+}(r)=n_{a}^{+}(0) J_{0}\left(\frac{2.4 r}{R}\right),
$$

where $J_{0}$ is the Bessel function of order zero, and $n_{a}^{+}(0)$ the density of the ions in the axis of the tube $\left.(r=0)^{*}\right)$.

*) The ion density of the admixture in the axis of the discharge tube $n_{a}+(0)$ appearing in formula (25) should not be confused with the density of the admixture gas at the cathode denoted by $n_{a}(0)$ appearing e.g. in formula (18). 
However, we shall use here a usual approximation, namely the parabolic distribution ${ }^{23}$ ):

$$
n_{a}^{+}(r)=n_{a}^{+}(0)\left(1-\frac{r^{2}}{R^{2}}\right) .
$$

This formula supplies also the approximation that $n_{a}^{+}=0$ at the wall. At pressure of a few torr this approximation can be made. For $n_{a}^{+}(r)$ we shall use the distribution given in formula (26).

$b$. The radial distribution of the gas temperature. The transport parameters $\mu_{a}^{+}, D$ and the axial gradient $(\mathrm{d} / \mathrm{d} x) n_{a}$ depend on the gas temperature $\left.{ }^{24}\right)^{25}$ ). The gas temperature is a funtion of $r$ and thus

$$
\mu_{a}^{+}=\mu_{a}^{+}(T(r)), D=D(T(r)) \text { and } \frac{\mathrm{d}}{\mathrm{d} x} n_{a}=\frac{\mathrm{d}}{\mathrm{d} x} n_{a}(T(r)) .
$$

For the determination ${ }^{23}$ ) of the function $T(r)$ we assume the parabolic charge distribution as given in formula (26). The heat dissipation $W(r)$ is proportional to the electron density:

$$
W(r)=W(0)\left(1-\frac{r^{2}}{R^{2}}\right) .
$$

The equation for heat transport in the cylindrical case is:

$$
\frac{\mathrm{d}^{2}}{\mathrm{~d} r^{2}} T(r)+\frac{1}{r} \frac{\mathrm{d} T(r)}{\mathrm{d} r}+\frac{W(0)}{\kappa}\left(1-\frac{r^{2}}{R^{2}}\right)=0,
$$

with $\boldsymbol{\kappa}$ as the coefficient of thermal conductivity and $W(0)$ as the energy dissipation per unit of volume in the axis of the tube. The solution of equation (28) is

$$
T(r)=T(R)+\frac{3}{16} \frac{W(0) R^{2}}{\kappa}\left\{1-\frac{4}{3}\left(\frac{r}{R}\right)^{2}+\frac{1}{3}\left(\frac{r}{R}\right)^{4}\right\} .
$$

The temperature difference between the axis and the wall of the tube, $\Delta T \equiv T(0)-T(R)$, is found from formula (29):

$$
\Delta T=\frac{3}{16} \frac{W(0) R^{2}}{\kappa} .
$$

The average gas temperature $\overline{T(\gamma)}$ in the discharge is:

$$
\overrightarrow{T(r)}=T(R)+\Delta T \frac{2}{R^{2}} \int_{0}^{R}\left(1-\frac{4}{3}\left(\frac{r}{R}\right)^{2}+\frac{1}{3}\left(\frac{r}{R}\right)^{4}\right) r \mathrm{~d} r=T(R)+\frac{4}{8} \Delta T .
$$

The average gas temperature $\overline{T(r)}$ can be determined experimentally from the increase in pressure after switching on the discharge (assuming no axial dependence). 
c. The ion mobility $\mu_{a}^{+}$and the diffusion cocfficiont as a function of $r$. The pressure in the discharge tube is equal cverywhere. The transport parameters $\mu_{a}^{+}$and $D$ are functions of the gas temperature. To give the exact dependence of $\mu_{a}^{+}$and $D$ on $T$ is a very complicated procedure and is different for every gas combination. The interaction potential $\left.{ }^{24}\right)^{25}$ ) of the colliding particles determines the temperature dependence of the transport parameters. As an approximation for the temperature dependence of $\mu_{a}^{+}$(for rigid sphere collisions) at constant pressure and not too low $T$ we use

$$
\mu_{a}^{+}(T)=\mu_{0}^{+}\left(T / T_{0}\right)^{\frac{1}{2}}
$$

where $\mu_{0}^{+}$is the mobility at a gas temperature $T-T_{0}$ (e.g. at $0^{\circ} \mathrm{C}$ ).

As an approximation for the diffusion coefficient at constant pressure (and for rigid sphere collisions) we will use:

$$
D(T)=D_{0}\left(T / T_{0}\right)^{3},
$$

with $D_{0}$ as the diffusion coefficient for $T=T_{0}$. The dependence of $\mu_{a}^{+}$and $D$ on $r$ can be found by substituting formula (29) in (32) and (33).

d. The gradient $(\mathrm{d} / \mathrm{d} x) n_{a}$ as a function of $r$. Experiments $\left.{ }^{*}\right)$ demonstrate that $(\mathrm{d} / \mathrm{d} x) n_{a} / n_{m}$ is a function of $x$ and does not depend on $r\left(n_{m}\right.$ is the density of the main gas). Since the pressure in the discharge tube is equal everywhere, the distribution of the density of the main gas is inversely proportional to the gas temperature in ${ }^{\circ} \mathrm{K}$ :

$$
n_{m}(T(r))=n_{m}(\overline{T(r)}) \cdot \overline{T(r)} / T(r) .
$$

The gradient $(\mathrm{d} / \mathrm{d} x) n_{a}(T(r))$ is given by:

$$
\left.\frac{\mathrm{d}}{\mathrm{d} x} n_{a}(T(r))=\overline{(T(r)} / T(r)\right) \cdot \frac{\mathrm{d}}{\mathrm{d} x} n_{u} \overline{(T(r))},
$$

with $T(r)$ and $\overline{T(r)}$ given in the formulas (29) and (31) respectively.

In the transport integrals in the formulas (22) and (23), the dependence of $\mu_{a}^{+}, n_{a}^{+}, D$ and $(\mathrm{d} / \mathrm{d} x) n_{a}$ on $r$ can be substituted. We will solve the integrals for a representative example of an experimentally derived value ${ }^{2}$ ) of $T(R)$ and $\Delta T$ and compare the result with the following approximative formulas for the transports:

$$
\begin{aligned}
& \pi R^{2} \overline{n_{a}^{+}(r)} \cdot \mu_{a}^{+}(\overline{T(r)}) E \text { ion transport, } \\
& \pi R^{2} D(\overline{T(r)}) \frac{\mathrm{d}}{\mathrm{d} x} n_{a} \text { diffusion transport. }
\end{aligned}
$$

Substitution of $T(R)=300^{\circ} \mathrm{K}$ and $\Delta T=50^{\circ} \mathrm{K}$ gives a result for the integral given in (22) that is $2 \%$ larger than that in formula (36). The difference

*) The experiments were carried out in the experimental arrangement to be described in our next paper ${ }^{2}$ ). 
between formula (23) and (37) is very small (1\%). The formulas (36) and (37) are much easier to handle and are a good approximation for (22) and (23) Thus, for the calculation in experiments one can use the formulas (36) and (37).

5. Time dependence of the segregation. When in a neutral, homogeneously distributed, gas mixture a discharge is switched on, the segregation in the gas mixture will build up. The time dependence of the distribution of the admixture gas along the discharge tube can be solved for gas mixtures with small amounts of admixture gas $\left(n_{a}^{+}=\theta n_{a}\right.$, with a constant $\left.\theta\right)$ from the diffusion equation:

$$
\frac{\partial}{\partial t} n_{a}(x, t)=D \frac{\partial^{2}}{\partial x^{2}} n_{a}(x, t)-u \frac{\partial}{\partial x} n_{a}(x, t),
$$

in which the second term of the right hand side accounts for the transport of the ions in the electric field. The solution of equation (38) with the initial conditions $n_{a}(x, 0)=n_{a}^{\star}$ for all $x$, and no gas losses at the ends of the tube is $\left.{ }^{26}\right)$ :

$$
\begin{aligned}
n_{a}(x, t)= & n_{a}(x, \infty)+n_{a}^{\star} B \exp \left[-\frac{u}{2 D} x-\frac{u^{2}}{4 D} t\right] . \\
& \sum_{n=1}^{\infty} C_{n}\left(\cos \frac{\pi n}{L} x-\frac{u L}{2 \pi D n} \sin \frac{\pi n}{L} x\right) \exp \left[-\frac{\pi^{2} D n^{2}}{L^{2}}\right],
\end{aligned}
$$

where

$$
\begin{aligned}
B & \equiv 32 D^{3} \pi^{2} L u \\
C n & \equiv\left(u^{2} L^{2} n^{-1}+4 \pi^{2} D^{2} n\right)^{-2}\left((-1)^{n} \exp \left[-\frac{u L}{2 D}\right]-1\right)
\end{aligned}
$$

and $n_{a}(x, \infty)$ is given in formula (19).

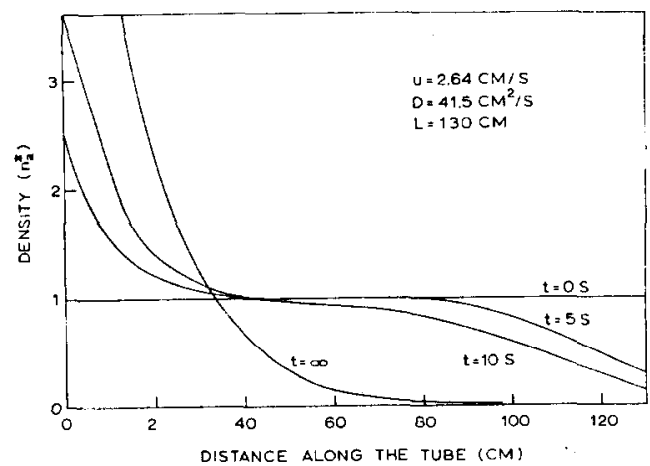

Fig. 1. The distribution of the density of the admixture along the positive column of a discharge for time in 5,10 and $\infty$ seconds. The distribution was calculated with formula (39), and is expressed in units of $n_{a}^{*}$ (the initial density). 
As an example we calculated the distribution along the positive column for certain values of $u, D$ and $L$ (see fig. 1). The values for $u, D$ and $L$ (given in fig. 1) were taken from experiments carried out in a neon-argon discharge 1) $\left.{ }^{2}\right)(0.2 \%$ argon; total reduced pressure is 6.76 torr $)$.

Acknowledgements. I wish to thank Prof. Dr J. A. Smit and Dr J. M. Fluit for their helpful discussions.

This work forms part of the research program of the "Stichting voor Fundamenteel Onderzoek der Materie" (F.O.M.), which is financially supported by the "Nederlandse Organisatie voor Zuiver Wetenschappelijk Onderzoek" (Z.W.O.).

Received 22-3-67

\section{REIERLNCLS}

1) Freudenthal, J., Thesis Utrecht (1966).

2) lircudenthal, J., Physica :B(; (1967) 365.

3) Skaupy, Ii., Verh. Phys. Gesells. 18 (1916) 230.

4) Skaupy, li. and Bobek, I., Z. techn. Phys. 6 (1925) 284.

5) Penning, k. M., Physica 1 (1934) 763.

6) Druyvestey 1, M. J., Physica 2 (1935) 255.

7) Krysmanski, K. H., Thesis Greifswald (1957).

8) Krysmanski, K. H., Ann. Physik 2 (1958) 263.

9) Schmelteliopf, A. L., Thesis Austin (1962).

10) Schmeltekopf, A. L., J. appl. Phys. 35 (1964) 1712.

11) Matveeva, N. A., Izv. Akad. Nauk. SSSR. Ser. Iiz (transl.) 23 (1959) 1009.

12.) Thomson, J. J., l'roe. roy. Soc. I.onkin 58 (1895) 244.

13) Kenty, C., Bull. Amer. Phys. Soc. Ser. 1I. 3 (1958) 82.

14) Locb, I.. B., J. appl. Phys. 29 (1958) 1369.

15) Strock, L. W., Spectrum analysis with the carbon are athode layer. Adam Hilger, Ltc. (London, 1936).

16) Nachtricb, H. N., Principles and practice of spectrochemical analysis. Mecraw-Hill Book Company, Inc. (New York, 1950).

17) Ahrens, I. H., Spectrochemical analysis, Addison-Wesley press, Inc. (Canbridur, Mass. USA, 1950).

18) De Galan, L., Thesis Amsterdan (1965).

19) Present, R. D., Kinctic theory of gases. McGraw-1 Lill Book Company, Ine. (Now York, 1958), esp. chapter 4.

20) Barbiere, D., Phys. Kev. 84 (1951) 653.

21) Loeb, I.. B., Basic processes of gaseous electronics. University of California presi (Burleley and Los Angelos, 1960).

22) Schottky, W., Phys. Z. 25 (1924) 635.

23) Kenty, C., Easly, M. A. and Barnes, B. T., J. appl. Phys. 22 (1951) 1006.

24) Mason, li. A. and Schamp, H. W., Ann. Phys. (New York) 4 (1958) 233.

25) Hirschfelder, J. O., Curtis, C. F. and Bird, R. B., Molecular theory of gases aud liquicls. John Wiley and Sons, Inc. (New York, 1954).

26) Fürth, R., Handbuch physik. Techn. Mech. 7 (Leipził, 1931) p. 635.

27) Rüttenauer, A., Z. Phys. 10 (1922) 269. 